\title{
Identification and characterization of Camellia sinensis glucosyltransferase, UGT73A17: A possible role in flavonol glucosylation
}

\author{
Shoji Ohgami ${ }^{1, \dagger}$, Eiichiro Ono ${ }^{2, \dagger}$, Hiromi Toyonaga ${ }^{2}$, Naoharu Watanabe $^{3}$, \\ Toshiyuki Ohnishi ${ }^{1,4, *}$ \\ ${ }^{1}$ Graduate School of Agriculture, Shizuoka University, Shizuoka, Shizuoka 422-8529, Japan; ${ }^{2}$ Research Institute, Suntory \\ Global Innovation Center Ltd., Mishima, Osaka 618-8503, Japan; ${ }^{3}$ Graduate School of Engineering, Shizuoka University, \\ Hamamatsu, Shizuoka 432-8561, Japan; ${ }^{4}$ Research Institute of Green Science and Technology, Shizuoka University, Shizuoka, \\ Shizuoka 422-8529, Japan \\ *E-mail: dtonish@ipc.shizuoka.ac.jp Tel \& Fax: +81-54-238-3082
}

Received October 6, 2014; accepted October 27, 2014 (Edited by A. Sugiyama)

\begin{abstract}
Tea plant (Camellia sinensis) biosynthesizes a wide variety of specialized metabolites, including phenolic compounds such as catechins. Flavonol, one of the major flavonoid subclasses, in $C$. sinensis is present in the $O$-glycoside form, such as quercetin 3-O- $\beta$-D-glucopyranoside, kaempferol 3-O- $\beta$-D-glucopyranoside, and rutin (quercetin 3-O- $\beta$ glucopyranosyl-6-O- $\alpha$-rhamnoside). These flavonol glycosides are highly accumulated, constituting up to $2-3 \%$ (w/w dry weight) of tea leaves; however, their biosynthetic machinery in C. sinensis remains elusive. Using high-throughput RNA sequencing from the fresh leaves of a cultivar (C. sinensis var sinensis cv Yabukita) and rapid amplification of cDNA ends (RACE) cloning with degenerate oligonucleotide primers, we identified a full-length cDNA of UDP-glycosyltransferase, designated as UGT73A17, and characterized the biochemical and molecular functions of UGT73A17. Recombinant UGT73A17 protein catalyzed 3-O-glucosylation of quercetin, yielding quercetin 3-O- $\beta$-D-glucopyranoside in vitro. The preferential expression of UGT73A17 gene in the mature, relative to young leaves, stems and roots, is roughly consistent with the accumulation pattern of flavonol glycosides in C. sinensis, suggesting that UGT73A17, in part, participates in the biosynthesis of flavonol glycosides in planta.
\end{abstract}

Key words: Camellia sinensis (tea plant), flavonoid, quercetin, specialized metabolism, UDP-glycosyltransferase.

Various tea products represented by green, oolong, and black teas are manufactured from the leaves of Camellia sinensis. A wide variety of flavonoid-class specialized metabolites, such as flavan-3-ols and flavonols are accumulated in the fresh leaves and processed tea products, and some have been shown to have beneficial activities for human health (Lin et al. 2003). Flavonols, a major subclass of flavonoids, are biosynthesized by a 2-oxoglutarate- $\mathrm{Fe}(\mathrm{II})$ dioxygenase superfamily enzyme, flavonol synthase (Lin et al. 2007) and accumulated as $O$-glycosylated forms (flavonol glycosides represented by rutin (quercetin 3-O- $\beta$-glucopyranosyl-6-O- $\alpha$ rhamnoside)) via glycosylation in $C$. sinensis, whereas flavan-3-ols (catechins and theaflavins) are present either in free forms or as gallic acid esters (Liu et al. 2012; Pang et al. 2013). Glycosylation of small molecules, such as specialized metabolites, alters their reactivity and stability, thereby regulating the cellular transport and storage of metabolites.

Uridine diphosphate (UDP)-sugar dependent glycosyltransferases (UGTs) are major enzymes transferring a sugar molecule from the sugar-donor, UDP-sugar, to acceptor molecules. UGT is one of the largest enzyme superfamilies, consisting of more than a hundred genes in a seed plant genome, (Bowles 2002). There are many reports of UGTs catalyzing the glycosylation of specialized metabolites e.g., grapevine (Vitis vinifera) has been known to have structurally similar but distinct UGT78A proteins catalyzing 3-O-glycosylation of anthocyanins and flavonols (Ford et al. 1998; Ono et al. 2010), whereas Petunia hybrida Rt (UGT79A1) has been known to catalyze 6"-O-rhamnosylation of glucose

Abbreviations: EST, expressed sequence tag; F3GlcT, flavonoid 3-O-glucosyltransferase; F3Glc-6-O-RhaT, flavonoid 3-O-glucose: 6-Orhamnosyltransferase; FLS, flavonol synthase; HPLC, high-performance liquid chromatography; qRT-PCR, quantitative reverse transcriptionpolymerase chain reaction; quercetin 3-O- $\beta$-glucopyranosyl-6-O- $\alpha$-rhamnoside, rutin; RACE, rapid amplification of cDNA ends; UGTs, Uridine diphosphate-sugar-dependent glycosyltransferases.

${ }^{\dagger}$ These authors contributed equally to this work.

This article can be found at http://www.jspcmb.jp/

Published online December 27, 2014 
<smiles>[R]c1cc(-c2oc3cc(O)cc(O)c3c(=O)c2O)cc([R])c1[R]</smiles>

flavonol

kaempferol: $\mathrm{R}_{1}=\mathrm{H} ; \mathrm{R}_{2}=\mathrm{OH} ; \mathrm{R}_{3}=\mathrm{H}$ quercetin: $\mathrm{R}_{1}=\mathrm{OH} ; \mathrm{R}_{2}=\mathrm{OH} ; \mathrm{R}_{3}=\mathrm{H}$ myricetin: $\mathrm{R}_{1}=\mathrm{OH} ; \mathrm{R}_{2}=\mathrm{OH} ; \mathrm{R}_{3}=\mathrm{OH}$<smiles>O=c1c(OC(CO)C(O)C(O)C(O)CO)c(-c2ccc(O)c(O)c2)oc2cc(O)cc(O)c12</smiles>

$$
\begin{aligned}
& \text { quercetin } 3-0-\beta-D- \\
& \text { glucopyranoside }
\end{aligned}
$$

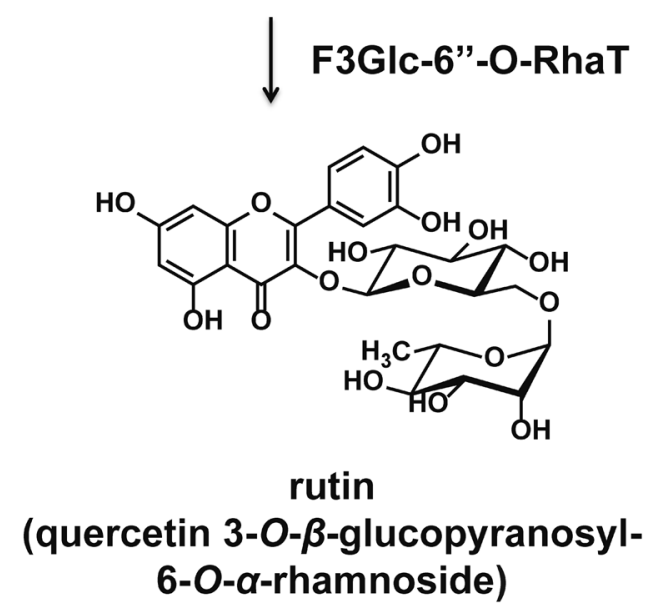

Figure 1. Putative biosynthetic pathway of rutin in Camellia sinensis. FLS: flavonol synthase, F3GlcT: flavonoid 3-O-glucosyltransferase, F3Glc-6-O-RhaT: flavonoid 3-O-glucose: 6-O-rhamnosyltransferase.

moiety of anthocyanin 3-O-glucoside, resulting in formation of anthocyanidin rutinoside (Brugliera et al. 1994).

Despite the high accumulation of flavonol glycosides [up to $2-3 \%$ (w/w dry weight) in tea products] and their useful biological activities (Lin et al. 2003), the biosynthetic machinery of flavonol glycosides in $C$. sinensis remains elusive. Here we present the molecular and biochemical characterization of a novel C. sinensis UDP-glucosyltransferase, UGT73A17, that catalyzes 3-Oglucosylation for flavonols (Figure 1).
To assess the distribution of flavonol glycosides in C. sinensis, the composition and content of flavonol glycosides in the young and mature leaves, stems, and roots were investigated. The fresh leaves, stems, and roots of tea plants were harvested at the Center for Education and Research of Field Sciences, Shizuoka University (Shizuoka, Japan). By following the same procedure described in Katsumoto et al. (2007), flavonoid fractions were extracted from the young and mature leaves, stems, and roots. Flavonol aglycones were quantitatively measured in enzymatically hydrolyzed fractions by highperformance liquid chromatography (HPLC), whereas flavonol glycosides were directly measured without hydrolysis. Larger amounts of flavonols were observed in the mature leaves and the young leaves, than in stems and roots (Figure 2). By hydrolysis of glycosides, quercetin was found to be the most abundant flavonol, rather than kaempferol and myricetin. Important to note, kaempferol was higher in the young leaves than in the mature leaves, whereas quercetin and myricetin were markedly increased in the mature leaves compared with those in the young leaves. Approximately twice the amounts of quercetin and its rutinoside (rutin) were found in the mature leaves of $C$. sinensis compared with those in the young leaves, suggesting that hydroxylation activity on the B-ring of flavonoids is activated along with leaf maturation. In contrast to rutin (a diglycoside), only small amount of quercetin monoglucoside was detected (data not shown), probably reflecting the metabolic coupling of 6 "-O-rhamnosylation on the glucose moiety of flavonol monoglucoside, with the first 3-O-glucosylation of flavonols in vivo. These results show a spatiotemporal change of flavonol glycosides in $C$. sinensis. In the aspect of the biological function that the mature leaves highly accumulated flavonols compared with those in the young leaves, the mature leaves of $\mathrm{C}$. sinensis may accumulate have phenolic compounds which protects the mesophyll tissue against UV-B radiation (280-315 nm) (Thompson et al. 1972).

To isolate candidate UGT genes involved in the glycosylation of flavonols in the leaves, we used transcriptome data derived from the sequencing of expressed sequence tag (EST) libraries. Total RNA was extracted from the young leaves of $C$. sinensis using a Plant RNeasy extraction kit (Qiagen, Tokyo, Japan) according to the manufacturer's instructions. Construction of cDNA libraries for Roche-GSFLX Titanium 454 sequencing (Roche Diagnostics, Tokyo, Japan), sequencing, trimming, and assembly of sequences were outsourced to Dragon Genomics Center (Mie, Japan) at TaKaRaBio Inc. In the resulting de novo transcript assembly from 454 sequencing, 187 independent ESTs assigned as UDP-glucosyltransferases by EST Viewer version 1.12 (TaKaRaBio) were obtained. Using TBLASTX search (Altschul et al. 1997), we 
A)

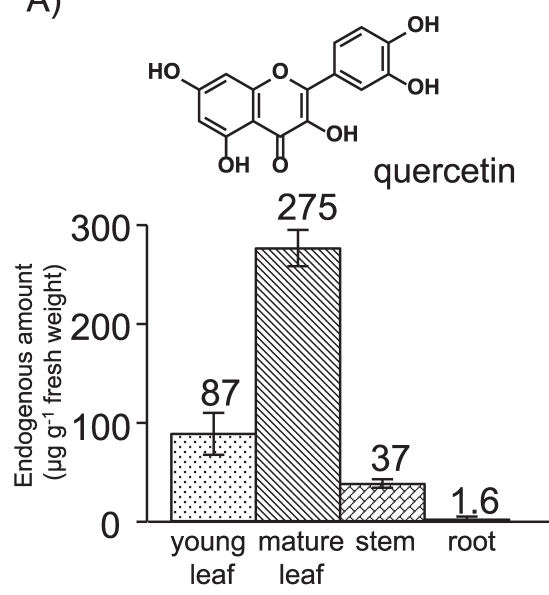

C)<smiles>O=c1c(O)c(-c2ccc(O)cc2)oc2cc(O)cc(O)c12</smiles>

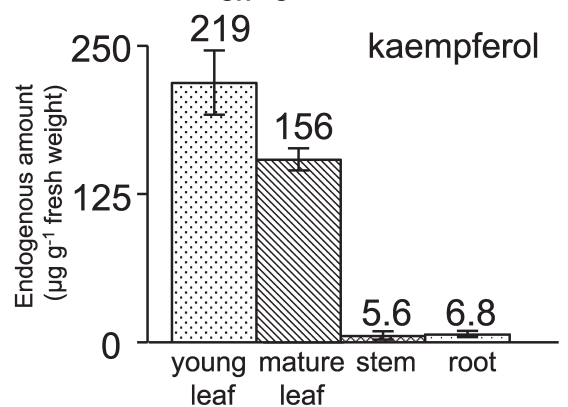

B)

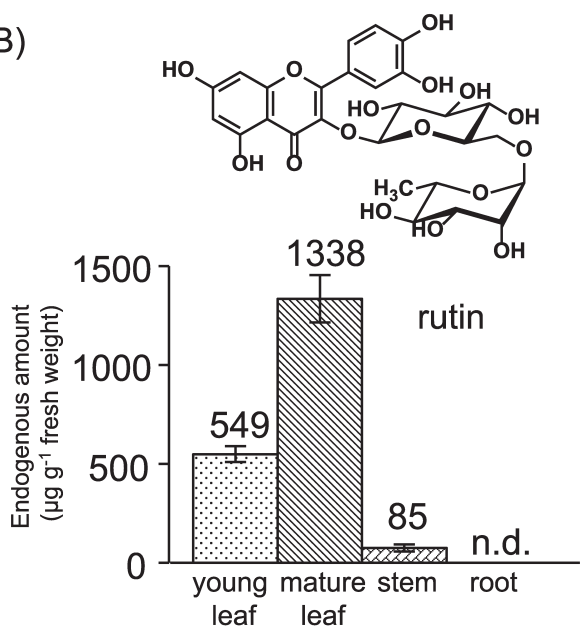

D)<smiles>Oc1ccc(-c2ccc(O)c(O)c2)c(O)c1</smiles>

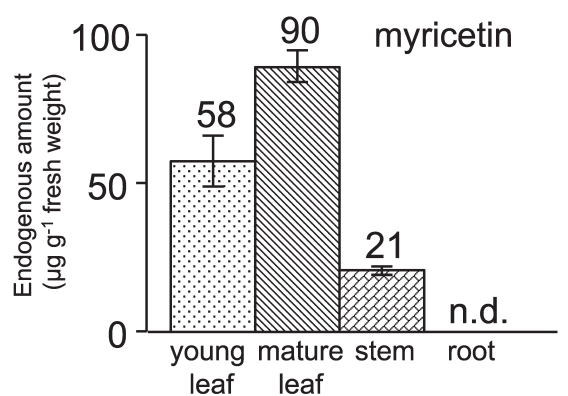

Figure 2. Quantitative analysis of endogenous flavonols and quercetin glycoside (rutin) in various organs of C. sinensis cv. Yabukita. A) quercetin, B) rutin, C) kaempferol, D) myricetin. Values were determined in three biological replicate measurements, error bars indicate standard deviation. n.d. means not detected by HPLC analysis.

screened these assembled ESTs for structural similarity to previously characterized flavonoid UGTs, such as flavonoid 3-O-glycosyltransferases (F3GlcT) and found 8 candidate UGT genes. Among the candidates, contig 2948 was found to have an incomplete open reading frame, and rapid amplification of cDNA ends to obtain a full-length cDNA of contig 2948 was performed using RACE primers (GR-Cs85AlikeC1-RV: 5'-CGA TCT TGC CTT TCC TCA CAA CCC AGA TG-3', and Cs85AlikeC1nest-RV: 5 ' -CTC AAC CTC ATC AAT GGC TGA TTC TTT AC- $3^{\prime}$ ) according to the procedure described previously (Ono et al. 2006).

The isolated full-length cDNA consists of a 1,422-bp open reading frame encoding a polypeptide of 473 amino acid residues (calculated molecular weight: $52.9 \mathrm{kDa}$ ) showing $78 \%$ structural similarity with a Fragaria vesca flavonoid: 3-O-glucosyltransferase (accession XM_004304666) and a Vitis vinifera flavonoid: 3-Oglucosyltransferase (accession XM_002282427). PSORT (http://psort.ims.u-tokyo.ac.jp/) analysis predicted that the isolated full-length cDNA harbored a conserved domain (W343-Q386; 44 amino acids), with UDPglycosyltransferase. This contig 2948 was designated as UGT73A17 (accession AB847095.1) by the committee responsible for naming UDP-glucuronosyltransferases (Mackenzie et al. 1997).

We assessed the biochemical activity of UGT73A17 using the recombinant proteins in vitro, according to procedures described in previous reports (Noguchi et al. 2009; Ono et al. 2010). In order to express UGT73A17 protein heterologously in Escherichia coli, cDNA of full-length UGT73A17 was amplified with NdeI and BamHI restriction sites using the primer set (CAC C-NdeI-CsUGT-C1-FW: 5' -CACCCA TAT GGC TAA GCT TCA TTT CTT C-3' and CsUGT-C1-BamHIstop-RV: 5 '-GGA TCC TTA TGA ACT CAT TTC TTG TATCAGAG-3'), ligated to the NdeI and BamHI sites in a pET15b expression vector (Novagen, Tokyo, Japan), and the ligated plasmid was transformed into E. coli BL21 (DE3) (TOYOBO, Osaka, Japan) after sequence confirmation. The recombinant UGT73A17 proteins were partially purified with an $\mathrm{N}$-terminal 
A)

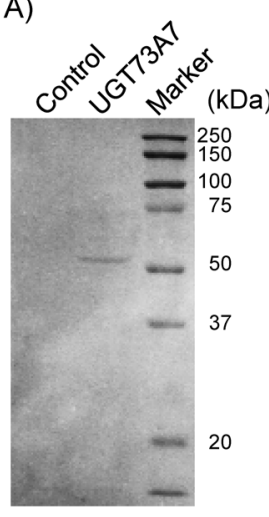

C)

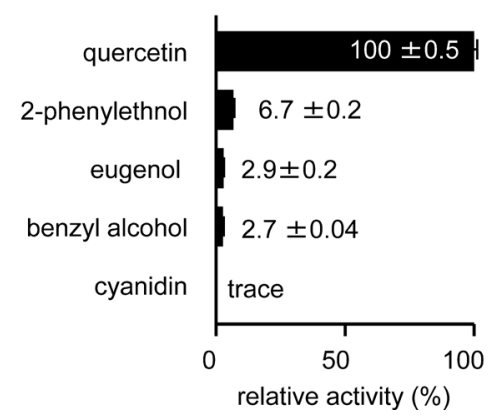

B)

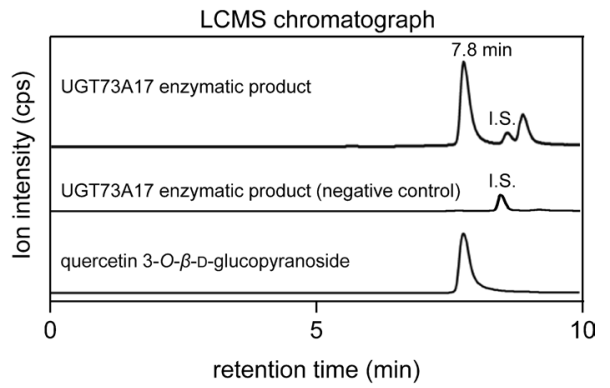

D)

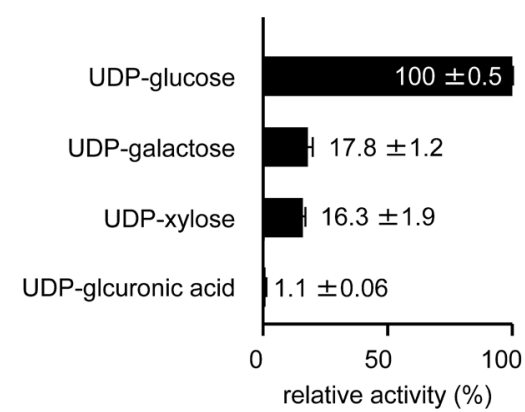

Figure 3. Biochemical characterization of UGT73A17. A) western blotting (left) and CBB staining (right) of heterologous expressed UGT73A17. B) LC-MS analysis of the enzymatic product of UGT73A17 with quercetin. Dnatureing UGT73A17 protein by heat-treatment $\left(90^{\circ} \mathrm{C}, 20 \mathrm{~min}\right)$ was used as negative control. LC-MS analysis was performed the following condtions; HPLC colomn; capcell pak column $\mathrm{C}_{18}$ UG120 (2.0 mm I.D. $\times 150 \mathrm{~mm}$, Shiseidou, Tokyo, Japan), Column temp.; $40^{\circ} \mathrm{C}$, mobile phase A: $0.05 \%(\mathrm{v} / \mathrm{v})$ formic acid in water, mobile phase B: acetonitrile, gradient condition; $15-51 \%$ of mobile phase B $(0-10 \mathrm{~min}$ ); flow rate; $0.2 \mathrm{ml} / \mathrm{min}$, ESI-MS (negative) monitoring; $\mathrm{m} / \mathrm{z} 463$ for quercetin 3-O- $\beta$-D-glucopyranoside and $\mathrm{m} / z$ 503 for eugenyl $\beta$-primeveroside $\left([\mathrm{M}+\mathrm{HCOO}]^{-}\right)$used as an Internal started (I.S.). C) Relative activity of UGT73A17 toward sugar acceptors (eugenol, benzyl alcohol, 2-phenylethanol, quercetin, and cyanidin). D) Relative activity of UGT73A17 toward sugar donors (UDP-glucose, UDP-xylose, UDPgalactose, and UDP-glucuronic acid). Values of relative activity of UGT73A17 toward sugar donors and sugar acceptors were determined in three replicate measurements, error bars indicate standard deviation.

His-tag (Figure 3A) and tested with UDP-glucose as a sugar donor against quercetin as previously described in Noguchi et al. (2009). The expressed recombinant proteins in SDS-PAGE gels were also immunologically confirmed by western blotting analysis with His.Tag monoclonal antibody (mouse, Novagen) and ECL Anti-mouse IgG Horseradish Peroxidase-linked Whole antibody (sheep, GE Healthcare, Tokyo, Japan), respectively. (Figure 3). The enzymatic assays were performed using the purified protein, sugar acceptor, and UDP-sugar in $50 \mathrm{mM}$ potassium phosphate buffer. The enzymatic reaction was stopped by addition of the same volume of methanol prior to LC-MS analysis. The apparent $K_{\mathrm{m}}$ and $V_{\max }$ values for glycosyl donors and the sugar acceptor (quercetin) in the presence of a saturating concentration of the counter substrate were determined by fitting the initial velocity data to the MichaelisMenten equation using nonlinear regression analysis with Kaleidagraph (Synergy Software, PA, USA).

In LC-MS analysis, two newly formed peaks in the reaction of quercetin with UGT73A17 were found at the retention time of $7.8 \mathrm{~min}$ and $8.8 \mathrm{~min}$ with $\mathrm{m} / \mathrm{z}$ 463. The dominant peak at $7.8 \mathrm{~min}$ was consistent with formation of quercetin 3-O- $\beta$-D-glucopyranoside (Figure $3 \mathrm{~B})$. Although we could not determine the chemical structure of the minor peak at the retention time of $8.8 \mathrm{~min}$, it has been reported that Arabidopsis UGT73B1 (At4g34138) catalyzes 7-O-glucosylation of quercetin (Kim et al. 2006). UGT73A17 may be a bifunctional UGT forming quercetin 3-O- $\beta$-D-glucopyranoside and quercetin $7-O-\beta$-D-glucopyranoside The maximum velocity $\left(V_{\max }\right)$ and estimated apparent $K_{\mathrm{m}}$ values of UGT73A17 with quercetin were determined to be $143.7 \pm 9.7 \mu \mathrm{mol} \mathrm{min}^{-1} \mathrm{mg}^{-1}$ protein and $9.9 \pm 2.3(\mu \mathrm{M})$, respectively. Two further flavonoids (quercetin and cyanidin) and three aromatic alcohols were tested as potential substrates. In contrast to its high activity toward quercetin (100\%), UGT73A17 showed negligible activity toward 2-phenylethanol (6.7\%), eugenol (2.9\%) and benzyl alcohol (2.7\%) (Figure 3C). Trace activity toward cyanidin was observed in spite of the structural similarity to quercetin. To evaluate the sugar donor specificity of UGT73A17, we tested four UDP-sugars (UDP-glucose, UDP-galactose, UDP-xylose, and UDP-glucuronic acid), with quercetin as a sugar acceptor. The relative activity of UGT73A17 toward UDP sugars was estimated as 100\% 
for UDP-glucose, $17.8 \%$ for UDP-galactose, $16.3 \%$ for UDP-xylose, and 1.1\% for UDP-glucuronic acid (Figure 3D). Taken together, these data showed that UGT73A17 preferentially catalyzes $3-O-\beta$-glucosylation of quercetin, with narrow substrate specificity in vitro.

The spatial gene expression of UGT73A17 was investigated by quantitative reverse transcriptionpolymerase chain reaction (qRT-PCR), with each organ of $C$. sinensis (young leaf, mature leaf, stem, and root) according to the procedure described by Noguchi et al. (2008), with the following specific primer sets: qRTCsUGT_C1-FW: 5' -TCA GCC ATT GAT GAG GTT GA and qRT-CsUGT_C1-RV: 5' -ATG AAT TGT TGC CCT GAA GC for UGT73A17, and Cs18SrRNA-FW: 5'CACGGG GAG GTA GTG ACA AT-3' and Cs18SrRNARV: $5^{\prime}$-CCT CCA ATG GAT CCT CGT TA-3' for $18 \mathrm{~S}$ rRNA of C. sinensis (accession number; AB120309) as an internal standard gene. Real-time PCR was performed using a 7500 Real-Time PCR system (Life Technologies) and a Power SYBR Green PCR kit (Qiagen). Relative transcription levels were analyzed by the DD cycle threshold method (Life Technologies) after normalization to expression of an internal standard (18S $r D N A)$.

UGT73A17 gene was found to be expressed at high abundance in the mature leaves (Figure 4). Along with leaf maturation, its expression increased, which is in accordance with the accumulation profile of quercetin glycoside (Figure 2). These results suggest that UGT73A17 partly participates in flavonol glycosylation in the leaves of C. sinensis.

This is the first report of $C$. sinensis UGT catalyzing glucosylation of flavonols. Owing to the reporting of various substrates as sugar acceptors for UGT73-family enzymes (Fukuchi-Mizutani et al. 2003), it is difficult

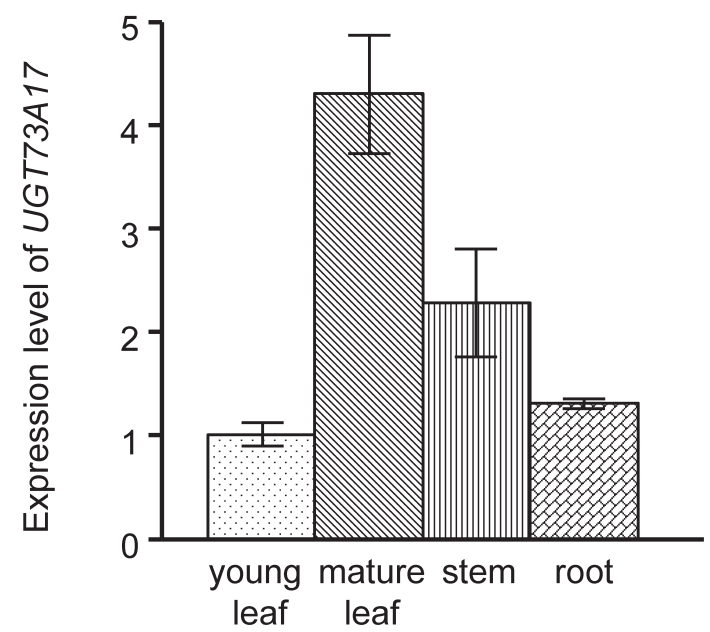

Figure 4. Relative transcript abundance of UGT73A17 in various organs (young leaves, mature leaves, stems, and roots) in C. sinensis. Expression level of UGT73A17 in young leaf is set to 1.0. Values of relative expression level were determined in three biological replicate measurements, error bars indicate standard deviation. to identify the bona fide substrates of enzymes of this family on the basis of phylogenetic relationships and in vitro characterization. However, our findings on the preferential expression of UGT73A17 in mature leaves, where rutin highly accumulates, and the catalysis of the recombinant protein for quercetin suggest that flavonols are potent substrates of this protein in vivo.

Given that rutin is the most abundant form of flavonol in leaves of C. sinensis (Figure 2), the second UGTs catalyzing 6"-O-rhamnosylation for glucose moiety of qurcetin 3-O-glucoside should be active for efficient conversion of qurcetin 3-O-glucoside to rutin (Figure $1)$. Among the 8 contigs showing structural similarity to UGT described above, we found contig2937 to be a candidate base on the homology to Petunia hybrida UGT79A1, which catalyzes 6"-O-rhamnosylation of anthocyanidin 3-O- $\beta$-glucoside (Brugliera et al. 1994; Kroon et al. 1994). Future functional characterization of contig2937 and UGT73A17 in association with flavonol synthase in vivo will clarify their biological impacts on tea flavonoid metabolism.

\section{Acknowledgements}

We thank Akiko Saito (Suntory) for technical support for this work. This study was supported in part by the JSPS KAKENHI program (grant numbers 26870252 to T.O)

\section{References}

Altschul SF, Madden TL, Schaffer AA, Zhang J, Zhang Z, Miller W, Lipman DJ (1997) Gapped BLAST and PSI-BLAST: A new generation of protein database search programs. Nucleic Acids Res 25: 3389-3402

Brugliera F, Holton TA, Stevenson TW, Farcy E, Lu C-Y, Cornish C (1994) Isolation and characterization of a cDNA clone corresponding to the Rt locus of Petunia hybrida. Plant J 5: 81-92

Fukuchi-Mizutani M, Okuhara H, Fukui Y, Nakao M, Katsumoto Y, Yonekura-Sakakibara K, Kusumi T, Hase T, Tanaka Y (2003) Biochemical and molecular characterization of a novel UDPglucose: anthocyanin 3 '-O-glucosyltransferase, a key enzyme for blue anthocyanin biosynthesis from gentian. Plant Physiol 132: 1652-1663

Katsumoto Y, Fukuchi-Mizutani M, Fukui Y, Brugliera F, Holton TA, Karan M, Nakamura N, Yonekura-Sakakibara K, Togami J, Pigeaire A, et al. (2007) Engineering of the rose flavonoid biosynthetic pathway successfully generated blue-hued flowers accumulating delphinidin. Plant Cell Physiol 48: 1589-1600

Kim JH, Kim BG, Park Y, Ko JH, Lim CE, Lim J, Lim Y, Ahn JH (2006) Characterization of flavonoid 7-O-glucosyltransferase from Arabidopsis thaliana. Biosci Biotechnol Biochem 70: 1471-1477

Kroon J, Souer E, de Graaff A, Xue Y, Mol J, Koes R (1994) Cloning and structural analysis of the anthocyanin pigmentation locus Rt of Petunia hybrida: characterization of insertion sequences in two mutant alleles. Plant J 5: 69-80

Lin YS, Tsai YJ, Tsay JS, Lin JK (2003) Factors affecting the levels of tea polyphenols and caffeine in tea leaves. J Agric Food Chem 51: $1864-1873$ 
Lin GZ, Lian YJ, Ryu JH, Sung MK, Park JS, Park HJ, Park BK, Shin JS, Lee MS, Cheon CI (2007) Expression and purification of Histagged flavonol synthase of Camellia sinensis from Escherichia coli. Protein Expr Purif 55: 287-292

Liu Y, Gao L, Liu L, Yang Q, Lu Z, Nie Z, Wang Y, Xia T (2012) Purification and characterization of a novel galloyltransferase involved in catechin galloylation in the tea plant (Camellia sinensis). J Biol Chem 287: 44406-44417

Mackenzie PI, Owens IS, Burchell B, Bock KW, Bairoch A, Bélanger A, Fournel-Gigleux S, Green M, Hum DW, Iyanagi T, et al. (1997) The UDP glycosyltransferase gene superfamily: Recommended nomenclature update based on evolutionary divergence. Pharmacogenetics 7: 255-269

Noguchi A, Fukui Y, Iuchi-Okada A, Kakutani S, Satake H, Iwashita T, Nakao M, Umezawa T, Ono E (2008) Sequential glucosylation of a furofuran lignan, (+)-sesaminol, by Sesamum indicum UGT71A9 and UGT94D1 glucosyltransferases. Plant $J$ 54: $415-427$

Noguchi A, Horikawa M, Fukui Y, Fukuchi-Mizutani M, IuchiOkada A, Ishiguro M, Kiso Y, Nakayama T, Ono E (2009)
Local differentiation of sugar donor specificity of flavonoid glycosyltransferase in Lamiales. Plant Cell 21: 1556-1572

Ono E, Nakai M, Fukui Y, Tomimori N, Fukuchi-Mizutani M, Saito M, Satake H, Tanaka T, Katsuta M, Umezawa T, et al. (2006) Formation of two methylenedioxy bridges by a Sesamum CYP81Q protein yielding a furofuran lignan, (+)-sesamin. Proc Natl Acad Sci USA 103: 10116-10121

Ono E, Homma Y, Horikawa M, Kunikane-Doi S, Imai H, Takahashi S, Kawai Y, Ishiguro M, Fukui Y, Nakayama T (2010) Functional differentiation of the glycosyltransferases that contribute to the chemical diversity of bioactive flavonol glycosides in grapevines (Vitis vinifera). Plant Cell 22: 2856-2871

Pang Y, Abeysinghe IS, He J, He X, Huhman D, Mewan KM, Sumner LW, Yun J, Dixon RA (2013) Functional characterization of proanthocyanidin pathway enzymes from tea and their application for metabolic engineering. Plant Physiol 161: $1103-1116$

Thompson WR, Meinwald J, Aneshansley D, Eisner T (1972) Flavonols: pigments responsible for ultraviolet absorption in nectar guide of flower. Science 177: 528-530 